\title{
IDONEIDAD DE LOS RANKINGS UNIVERSITARIOS
}

\section{Los rankings universitarios como parte del sistema de educación superior}

En un ambiente de multiplicación de la oferta de programas de educación superior, caracterizado por la ausencia de controles públicos o estatales de todo tipo y una regulación jurídica que inhibe la existencia de formas de control o escrutinio social institucionalizado, un sistema de información al usuario resulta indispensable.

El fundamento es que el producto que se ofrece presenta una complejidad mayor para el usuario, pues éste carece de los conocimientos adecuados para poder dirimir entre una buena o mala oferta educacional. La mayoría de los estudiantes que postulan a la universidad y sus padres no están en condiciones de elegir conforme a específicos estándares de calidad lo que más conviene a sus intereses. Cómo saber si se está ante una buena escuela de Derecho, de Medicina o de Ingeniería no es una pregunta que se pueda responder apreciando sólo los edificios, el número de computadores por alumno o los campos deportivos de que dispone la institución, ni siquiera los montos de matrícula o aranceles que cobran pueden ser descriptores confiables para tal decisión. Al contrario, todos estos ingredientes pueden actuar como eficaces factores de distracción u ocultamiento de aquello que realmente interesa saber.

Por la misma razón, la existencia de un agente institucional -sea de origen privado, estatal o mixto- que emita evaluaciones periódicas constituye una intervención externa del ajuste espontáneo y directo que cabe esperar de una relación ecuánime del usuario con el oferente. Desde este punto de vista adquiere pleno sentido y relevancia analizar los efectos que esta intervención puede producir. 
En principio, todo escrutinio destinado a generar información pública representa una ventaja, en cuanto dota al interesado de elementos de juicio relevantes para decidir sus opciones de estudio y calificación futura para el mercado laboral. Pero su orientación se construye sobre la base de dar prioridad a determinadas variables o criterios de medición, y de considerar ciertas fuentes de información, así como de decisiones metodológicas que, razonablemente, interfieren en los resultados y conclusiones de la jerarquización que todo ranking supone.

Podemos decir que, según sea el medio ambiente social y cultural en que nos hallemos, será también mayor o menor el grado de intervención o de influencia de la información producida en el sistema de educación superior. Si el contexto del mercado educacional es opaco, lo que está determinado por factores socioculturales nada misteriosos, la influencia de los rankings puede ser decisiva. De esto también se puede colegir que su sola existencia no contribuye a dar mayor transparencia al sistema. A mi juicio, resulta evidente que en un país como Chile, en que el $70 \%$ del ingreso a la universidad corresponde a jóvenes cuyos padres carecen de estudios superiores, la importancia de un sistema de información sobre prestigio u otra evaluación puede llegar a ser determinante en las preferencias y, con ello, inclinar a legitimar cierto tipo de universidades por sobre otras. En otras palabras, la orientación que los rankings puedan entregar -si son acogidos por la población- progresivamente determinará una cultura que luego influirá sobre las necesidades de sobrevivencia y sustentabilidad de los modelos de universidad socialmente demandados.

Con estas razones, podemos afirmar que, en la medida en que los rankings constituyen un entorno de información que genera y modela expectativas sobre los usuarios y, luego, sobre las instituciones de educación superior, forman parte de la estructura del sistema universitario. No es necesario suscribir funcionalismo teórico alguno para interpretar el contexto en que debemos analizar la importancia de los rankings de universidades. 


\section{Condiciones básicas o preliminares de un ranking universitario}

De la misma argumentación y conclusión precedentes surgen otras consecuencias dignas de analizar, tales como:

- ¿Qué condiciones mínimas de calidad deben cumplir a su vez las metodologías de medición de la calidad en un sistema universitario determinado?

- ¿Cuál es la garantía de independencia e imparcialidad del agente emisor del ranking?

1. La presencia gravitante de criterios provenientes del mundo académico

La calidad de las mediciones debería ser concebida en función de ciertos patrones determinados por la comunidad académica. Esta es la mejor fuente de legitimidad y ecuanimidad de los estándares e indicadores de una evaluación que conduce a un orden de prelación. Lo ideal sería que las propias universidades generaran un procedimiento autónomo, no estatal, o que las comunidades académicas estuvieran presentes en las diversas agencias emisoras para fijar las variables de medición.

La inexistencia de un ranking que nazca de las propias comunidades académicas o de las instituciones puede permitir intervenciones forzadas sobre el sistema de educación superior. Uno ajeno a aquellas, no obstante su capacidad de divulgación y notoriedad, podría poner en circulación una jerarquización que, al no ser reconocida por las instituciones, actúa sólo de cara al público, pero no impacta en la comunidad académica de modo alguno. Está claro que ningún ranking puede cumplir el rol que cabe a los procesos de acreditación, que han de tener mayor impacto en las instituciones que en el público, pero ello no es incompatible con los favorables efectos que un sistema de información debería también tener respecto de las propias instituciones y sus niveles de mejoramiento en la calidad del cumplimiento de sus fines académicos. 
Las cualidades de la medición no se reducen a que se halle inspirada en la presencia del mundo académico, pues comprenden un conjunto de factores que intentamos desarrollar a lo largo de este artículo.

\section{Independencia e imparcialidad}

La concurrencia de diversos agentes productores de rankings es una condición que debería favorecer la independencia e imparcialidad de las evaluaciones que llevan a cabo, pues el mismo argumento que justifica la necesidad de asistir al usuario para ponderar la calidad de las universidades y programas, esto es, su falta de elementos de juicio para una opción racional, también parece ser válido para que el usuario tenga acceso no a un solo emisor, sino a diversas fuentes de información.

La existencia de un sola fuente o agencia emisora de rankings, sea de carácter estatal o privado, es inconveniente para la legitimidad social de la tarea que deben cumplir. Por razones que sería fácil identificar lo mejor es que los usuarios dispongan de diversas fuentes que compitan entre sí por comunicar criterios objetivos, sobre la base de metodologías sancionadas por las comunidades académicas. Esto es particularmente relevante en todos los casos en que coexisten universidades públicas y privadas, o, al margen de dicha clasificación, cuando conviven instituciones nuevas que no son comparables con las tradicionales, públicas o privadas, o cuando -tercera hipótesis de diferenciación- un mismo sistema comprende universidades metropolitanas y regionales. La diversidad de agencias emisoras podría ayudar a reparar en los sesgos o inclinaciones de un ranking o a dejarlos de manifiesto, hecho que por sí solo contribuye a la necesaria transparencia ideológica y política de todo sistema de información pública.

Creemos conveniente por ello la constitución de un agente público, no estatal, que emita rankings del sistema educacional y coexista con otros de carácter privado. En este orden de cosas, nos parece indispensable la búsqueda de formas institucionales que 
no reduzcan lo público a lo estatal o gubernamental. Una fuente emisora de un ranking universitario que exprese una pluralidad de voluntades y concepciones no puede responder, en un ambiente social y políticamente complejo, a las ideas del gobierno de turno o a potestades puramente estatales. Estimamos que, con la serenidad y deliberación debidas, es perfectamente posible concebir una forma institucional que reúna y exprese la diversidad intelectual de un país y garantice la presencia de metodologías y perspectivas que emanen genuinamente del mundo académico.

Por lo mismo, la existencia exclusiva de fuentes privadas podría generar dos desventajas significativas relevantes: no garantizar la expresión de criterios académicos y que tales emisores no den a sus evaluaciones el grado de independencia e imparcialidad necesarios respecto de tendencias o de ciertos actores o instituciones del mismo sistema.

La elaboración de la metodología para jerarquizar las instituciones en razón de su prestigio o reputación requiere la intervención de académicos, cuyas opiniones han de ser determinantes. Esto debe ser así simplemente porque quienes están en condiciones de evaluar una actividad son precisamente aquellos que la conocen del modo más calificado. Resulta difícil admitir que los parámetros de calidad de un deporte sean establecidos por profesionales ajenos a éste; pues bien, la identificación de las cualidades ideales de una universidad y la comparación entre ellas no debe prescindir de quienes se dedican a la profesión académica. Esta, me parece, es la vía conveniente para no confundir los rankings de prestigio académico con encuestas de empleabilidad o válidas para ciertos efectos de desempeño profesional. Ciertamente, la existencia de emisores privados no es equivalente a prescindencia de criterios académicos; sin embargo, la libertad con que deben actuar y contar impide asegurar en todos los casos la sujeción rigurosa a dichos criterios.

La independencia e imparcialidad de las evaluaciones, especialmente en un medio cruzado por tensiones acerca de la función 
y carácter de las universidades, y de su relación con el Estado o con la empresa, resultan ser condiciones fundamentales para la legitimidad de tales evaluaciones. No es un secreto que los distintos proyectos sociales y políticos toman forma en materia de educación superior según opten por la promoción de instituciones privadas o la defensa de instituciones tradicionales o públicas. Desde este punto de vista, los rankings de origen exclusivamente privado que se asocian cultural o institucionalmente con las tendencias privatistas en educación tienden a un compromiso ciudadano con tales proyectos o a privilegiar, por sincera convicción, aquellos aspectos que les favorecen, pero sin que ello responda a reglas generalmente aceptadas en el medio académico.

\section{La inducción sintomática de preferencias y la transparencia del sistema}

No es necesario profundizar en el efecto persuasivo que pretende, y que en efecto tiene, todo ranking en cualquier orden de cosas. Sin embargo, tratándose de la educación superior es obvio que tal efecto compromete una cuestión de interés público que no puede quedar sustraída a la reflexión colectiva, y especialmente de la comunidad académica. La influencia debe ser ponderada tanto en relación con la masa de receptores y destinatarios como en consideración de los criterios utilizados para determinar las posiciones relativas que luego se asignan a las instituciones o programas.

Los destinatarios de las evaluaciones están expuestos mayoritariamente a una información que se construye luego de considerar una multiplicidad de datos, es decir, los resultados normalmente suponen un conocimiento de herramientas técnicas indispensables para su interpretación o decodificación, de cara a la decisión práctica y concreta que debe adoptar el postulante o quienes tienen influencia en esa decisión.

La capacidad del usuario para filtrar o controlar por sí mismo la validez de la información es y ha sido precaria en el sistema 
universitario chileno. Tal vez ello explica la expansión exitosa y en poco tiempo de la oferta en carreras y especialidades para las que el país, digámoslo, no disponía de una docencia de calidad o cercana siquiera a los niveles que se habían alcanzado en Chile después de un largo esfuerzo.

De esta verificación, nada se puede atribuir como responsabilidad a los rankings, lo que sí se puede inferir es que la demanda en Chile por educación superior no ha sido todo lo selectiva y exigente que uno pudiese desear. Este hecho, en consecuencia, sí demuestra que la interferencia en la transparencia y simetría de la información puede verse sustancialmente alterada por la emisión de jerarquizaciones que se constituyan como únicas y alcancen una amplia cobertura en el sistema.

En la medida en que tanto el bien ofrecido y evaluado requiere de un conjunto de conocimientos de alguna complejidad, su presentación está expuesta a distorsiones, consecuencia de la propia actividad de comparar a partir de creencias y conceptos no siempre validados por quienes ofrecerían mayor garantía de pertinencia y cuantía descriptiva. De este modo, los indicadores escogidos pueden omitir o asignar una baja ponderación a cualidades como los niveles de exigencia -claramente asociada a requisitos de selectividad del ingreso-, preferencia de los mejores puntajes, nivel de productividad científica e investigación, publicaciones, calidad de los académicos, recursos para la enseñanza y otras que inciden de modo directo en la calidad de la formación que las instituciones jerarquizadas pueden entregar.

En otras palabras, la sola existencia de publicaciones de rankings no constituye por sí misma una mejora de las condiciones para la decisión, pues también una proliferación de emisiones periódicas construidas sobre la base de indicadores poco fiables puede contribuir a la opacidad del sistema. 


\section{Los rankings por encuestas}

Los indicadores de percepción pueden formar parte de un conjunto de indicadores para establecer correlaciones entre diversas instituciones y ser, a la vez, un antecedente valioso para estas últimas, pero no pueden comportarse como matrices exclusivas de calificación. Las encuestas en que se considera a un universo de sujetos delimitados por su asociación a determinadas actividades empresariales, profesionales o públicas, y en que los criterios académicos no son de ordinaria aplicación, propenden a reproducir ciertos tópicos presentes en los discursos de mayor circulación, generando un circuito de autoafirmación de los mismos.

Esto puede ocurrir en los casos en que se producen jerarquizaciones sobre la base de entrevistas o consultas a profesionales destacados en una determinada actividad. La percepción del profesional podría, razonablemente, privilegiar aquellos aspectos que, desde su perspectiva en el mercado laboral, le parecen de la mayor significación, sin que esté en condiciones de ponderar o relevar aquellos que, de modo menos aparente, influyeron en su propia formación.

Además, el método que se basa en la medición de percepciones puede tender a la realización de una profecía autocumplida, por cuanto no hace más que satisfacer las expectativas socialmente ya constituidas según la precipitada ecuación o relación que se hace entre calidad en la formación y éxito profesional. En este caso, el ranking por encuesta tiende a ser la ratificación de una percepción preconstituida por hechos ajenos y no siempre relevantes para los objetivos de información objetiva que persigue el usuario.

No parece difícil de explicar que, en lo segundo, influyen factores de diverso carácter y para cuya debida comprensión se hace inevitable acudir al contexto en que se desenvuelve el sistema de educación superior. En la medida en que las elites políticas y económicas tienden a concebir utilitariamente la función de la universidad, se transita también a simplificar la tarea formadora de estas instituciones, la que debe reducirse a una docencia muchas 
veces toscamente entendida en el sentido de que no requiere más que transmitir ciertos conocimientos o destrezas perfectamente delimitadas. Por lo mismo, todas las instituciones que cumplen con esta función se hallan en condiciones de igualdad y la diferencia viene a estar dada por la opinión de los usuarios o de un tipo de éstos validados por esas mismas elites políticas y económicas. Esta concepción de la universidad es a su vez apoyada por un conjunto de acciones y recursos mediáticos ampliamente reconocidos e influyentes, que contribuyen a crear un entorno social y cultural predispuesto a acoger dogmáticamente la ecuación antes señalada.

Así, una buena universidad es aquella que asegura al estudiante buenas oportunidades en las empresas, de modo que los consultados de mayor interés resultan ser los gerentes de recursos humanos o jefes de personal. En este circuito se pierde la importancia que tienen las bibliotecas, la calidad de los claustros académicos, las exigencias de aprobación, calidad de los estudiantes, la investigación que se produce $\mathrm{u}$ otros factores de la mayor relevancia.

\section{Vaguedad de ciertas categorías de jerarquización}

Los llamados rankings de prestigio institucional constituyen quizás una de las metodologías que con más cuidado debemos analizar. El prestigio es una categoría que se construye sobre la base de generalizaciones derivadas de percepciones públicas, pero que se indagan a partir de preguntas que el investigador define y del cruce de información que el mismo controla.

Esto puede dar lugar a ciertas inconsistencias en la evaluación que no encuentran una explicación razonable a simple vista. Una misma fuente emisora de un ranking puede ubicar a una institución en un destacado lugar de prestigio, pero luego la misma institución desciende significativamente cuando se considera la preferencia de los mejores estudiantes que ingresan al sistema, o se atiende a la calidad de los profesores que forman el claustro. 
La distorsión puede alcanzar un alto grado; puede ocurrir que una institución como la Universidad de Chile en un ranking nacional se ubique en el segundo lugar de prestigio con promedio 6.53, seguida inmediatamente por la Universidad Adolfo Ibáñez con promedio 6.24 , pero que cuando se considera la preferencia de los mejores estudiantes por esas mismas instituciones se verifique que la primera se sitúa en el primer lugar con un $30.08 \%$ de los puntajes sobresalientes, mientras que la segunda, para este criterio, se halla en el lugar $14^{\circ}$ con $1,14 \%$ de los mejores estudiantes ${ }^{1}$. Es evidente que la propia fuente emisora del ranking deja constancia de la desproporción que se produce en esta variable específica pero fundamental. A simple vista, no parece explicable cómo pueden ambas ostentar una misma reputación o levemente diferenciada y, a la vez, ser percibidas de modo tan distinto por los estudiantes que, con seguridad, alcanzarán los mejores resultados académicos y profesionales. Lo que aparece del dato considerado es que los mejores estudiantes, por alguna razón distinta al prestigio de ambas, optan mayoritariamente por la Universidad de Chile; de mayor interés resulta todavía considerar que estos mismos estudiantes provienen de los sectores sociales más ilustrados y con elevado conocimiento del peso relativo de las instituciones de educación superior.

En el mismo sentido habría que destacar que, si se consideran los proyectos del Fondo de Desarrollo Científico y Tecnológico (Fondecyt), de tipo regular aprobados para el año 2006, la Pontificia Universidad Católica de Valparaíso aparece con 19 proyectos y la Universidad Diego Portales sólo con 5. Pues bien, cuando la misma fuente emisora considera estas universidades a propósito de la escala de prestigio las ubica en lugares sucesivos ( 7 y 8 respectivamente) y con una nota promedio de 5.42 y $5.22 .^{2}$ Luego, cuando se aprecian las preferencias de los mejores postulantes, el ranking muestra que la Pontificia Universidad Católica de Valparaíso capta 2.91\% y la Universidad Diego Portales el $0.63 \%$. En este caso hemos considerado

1 Ranking de revista Qué Pasa 2005, disponible en: http://www.noticias.utfsm.cl/prestigio05. pd, http://www.noticias.utfsm.cl/calidad_alumnos.pdt

2 Disponible en http://www.conicyt.cl/bases/fondecyt/index2.html\#institucion 
dos indicadores relevantes en que las diferencias son también enormes. Lo único que sugiere esta asimetría en cuanto al prestigio -que se convierte en convergencia en la llegada- es que la universidad favorecida está en Santiago y sus egresados acceden al mercado laboral más potente que existe en el país. Esto último provoca una distorsión asociada al desigual desarrollo económico que han alcanzado las principales ciudades de Chile, pero, a la vez, muestra cómo la categoría "prestigio o reputación de universidades" puede ocultar o subordinar indicadores relevantes para la decisión de un usuario.

Los argumentos anteriores permiten sugerir también que la reputación o prestigio no emana de fuentes informadas, lo que probablemente se explica porque las personas consultadas no integran las comunidades académicas y, específicamente, de académicos y docentes mayormente reconocidos. Un ranking de prestigio, como se dijo, no es un ranking de empleabilidad porque en el acceso al mercado laboral no influye solamente la calidad de la formación académica recibida, es decir, la institución donde realizó sus estudios. No se necesita enfatizar que el capital social y cultural que buscan las empresas no está determinado únicamente por el talento, las calificaciones y la institución académica de procedencia. Además, las mismas empresas tampoco dejan de ser vulnerables a otras presiones ajenas a las variables mencionadas, ni a signos de confianza y reconocimiento mutuo entre las personas que funcionan y registran al margen de reglas claramente formalizadas en la comunicación social, pero no menos eficaces.

Un ranking de prestigio institucional debe construirse sobre un conjunto de coordenadas conducentes a un juicio sobre la reputación académica de las instituciones evaluadas; tales coordenadas -entre otras- deberían ser: calidad de sus claustros, calidad de los alumnos, proyectos de investigación con financiamiento externo a la institución, recursos para la enseñanza y publicaciones. Estas variables pueden ser llevadas a cuantificadores bien fundados u objetivos, de modo que no dependen de percepciones u opiniones de personas ajenas a la actividad académica. 


\section{Equilibrio y coherencia de los indicadores}

Uno de los rankings consultados para este efecto, que busca precisamente establecer niveles de prestigio académico en el área de las escuelas de Derecho, es el que elabora el profesor Brian Leiter, de la Universidad de Texas. Recurre, en primer lugar, a la consulta de los 150 académicos más destacados en el ámbito de las ciencias jurídicas y busca, luego, establecer precisamente el nivel de reconocimiento que, entre ellos, se atribuye a los cuerpos docentes de las instituciones evaluadas. Pero también elabora otros rankings atendiendo a otros indicadores ${ }^{3}$.

La lectura que me interesa advertir es que en la tabla que sigue se consideran tres rankings distintos y cuya jeraquización, en consecuencia, responde a tres tipos de datos cualitativamente diversos, que emanan de fuentes diferentes. En la primera columna se ordenan las escuelas de Derecho en función de la calidad de sus cuerpos docentes, apreciada por la opinión de los académicos seleccionados para este mismo objetivo; en la segunda columna las escuelas por el nivel de preferencia del mejor cuarto de los postulantes en las pruebas de selección nacional; en la tercera columna, la jerarquización responde a una cuantificación del número de veces que son citados los académicos de esas mismas escuelas de Derecho. Si bien los datos se hallan relacionados, todos tienen un origen distinto y en los casos de las columnas dos y tres se trata de datos objetivos que constan de hechos atribuibles a terceros, no controlables por el emisor del ranking.

Ranking de escuelas de Derecho en Estados Unidos, realizado por el profesor Brian Leiter.

\begin{tabular}{llll}
\hline & 1. CALIDAD DEL CLAUSTRO & 2. CALIDAD DE ALUMNOS & 3. CLAUSTRO MÁS CITADO \\
\hline 1 & Yale & Harvard & Chicago \\
\hline 2 & Harvard & Yale & Yale \\
\hline 3 & Chicago & Columbia & Harvard \\
\hline 4 & Stanford & New York & Stanford \\
\hline 5 & Columbia & Chicago & UC Berkeley \\
\hline 6 & New York & Stanford & New York \\
\hline
\end{tabular}

3 http://www.leiterrankings.com 


\begin{tabular}{clll}
\hline 7 & UC Berkeley & Virginia & Columbia \\
\hline 8 & Michigan, Ann Arbor & Pennsylvania & Georgetown \\
\hline 9 & Texas & Northwestern & Duke \\
\hline 10 & Virginia & Georgetown & Texas, Austin \\
\hline 11 & Pennsylvania & Michigan, Ann Arbor & Cornell \\
\hline 12 & Georgetown & UC Berkeley & Northwestern \\
\hline 13 & Southern California & UCLA & Michigan, Ann Arbor \\
\hline 14 & Cornell & Duke & Virginia \\
\hline 15 & Northwestern & Texas, Austin & UCLA \\
\hline 16 & UCLA & Cornell & George Washington \\
\hline 17 & Duke & Fordham & Boston University \\
\hline 18 & Vanderbilt & Minnesota, Twin Cities & Colorado, Boulder \\
\hline 19 & Boston U. & Washington U, St. Louis & Emory \\
\hline 20 & Iowa & Illinois, Urbana-Champaign & Illinois, Urbana-Champaign \\
\hline
\end{tabular}

Aunque no explicaremos el criterio de selección de los académicos cuyas opiniones determinan la jerarquización que figura en la primera columna de nuestro gráfico, es evidente que ello introduce en esta evaluación un componente que no se puede calificar como plenamente objetivo. En todo caso, no resulta para nada despreciable que esta sea la opinión de los pares o expertos del sistema de formación jurídica en Estados Unidos, dato del que no se puede prescindir por lo que ya hemos afirmado en este texto. No se puede preguntar sino a quienes se han dedicado a enseñar Derecho. En la segunda y tercera columnas, como se dijo, los datos refieren a decisiones de terceros que constan objetivamente.

Lo interesante es que si apreciamos los lugares en que se ubican las universidades en las tres columnas, nos encontramos con que ellas no modifican sustancialmente sus posiciones relativas. En tal sentido me parece útil destacar las siguientes observaciones:

a) Casi todas las escuelas consideradas muestran niveles semejantes en los tres indicadores, de modo que las mejores 20 según los pares evaluadores lo son también si su opinión se contrasta con hechos ajenos a sus opiniones.

b) Los mejores estudiantes, de modo independiente, eligen las escuelas que cuentan con los cuerpos docentes más calificados, según el juicio de los académicos destacados en Estados Unidos. 
c) Los mejores estudiantes, de modo independiente, eligen las escuelas donde dictan clases los académicos más citados en los textos jurídicos que se producen en Estados Unidos.

En otras palabras, los estudiantes norteamericanos de la carrera de Derecho coinciden con los académicos más destacados de su país, y unos y otros coinciden, a su vez, con una comunidad de investigadores mucho más amplia y ajena. Este hecho no puede ser trivial, pues el punto de partida de la preferencia es cualitativamente diferente $y$, sin embargo, se observa una simetría admirable en los resultados.

El ejercicio analítico busca mostrar que la mejor manera de validar un ranking de prestigio o reputación es arribar a éste luego de cruzar resultados que provienen de indicadores diferentes. De esta manera se aprecia con nitidez que los mejores alumnos suelen elegir las mejores universidades y que éstas lo son precisamente porque son elegidas por lo más selecto del universo que postula. Este mismo procedimiento permite validar que las opiniones de pares académicos no se apartan de las preferencias de postulación, ni de la valoración que hace la comunidad científica, precisamente porque son pares calificados y destacados.

Por último, considero relevante señalar que en estos tres rankings no se producen las distorsiones que antes analizamos debido, en gran medida, a que la calificación se hace desde el propio sistema de educación superior, en este caso el de las escuelas de Derecho de Estados Unidos, a partir de sus recursos y actores, sin hacer intervenir elementos ajenos a la universidad. En todo caso, no sería razonable omitir que el caso analizado corresponde a un ambiente estabilizado, sin las tensiones ni transiciones que podemos observar en el sistema universitario chileno. 


\section{Pluralidad de criterios para rankings nacionales de instituciones}

No participo de la idea de cuestionar la utilidad de las jerarquizaciones, pues cumplen una función necesaria para llamar la atención pública sobre la calidad de los estudios superiores y promueven un debate público ampliado que, de otro modo, quedaría reservado a los sectores más cultivados. Además, en una sociedad en que se expande el acceso a la educación superior y se multiplican las instituciones que la imparten, el ranking satisface una necesidad de información que ya no puede circular del modo tradicional.

Que existan elementos subjetivos al momento de seleccionar información y cruzar variables de datos no parece una objeción sólida ya que, en un medio de libertad y pluralismo, los emisores tienden a autorregularse. Sin embargo, ello no obsta para que los emisores de tales rankings cuiden las metodologías que les sirven de base, pues ello aumentará la eficacia de sus pretensiones sociales. Un buen recaudo en tal sentido es que en aquellos en que se busca jerarquizar por calidad o por prestigio, que parece una forma indirecta de sugerir calidad, se utilicen diversos datos que emanen de fuentes diferenciadas y que consten de hechos objetivos o cuantificables, o de decisiones de terceros que se adoptan de modo independiente.

Para razonar sobre estas cualidades consideraré un ranking de las universidades españolas que lleva a cabo el diario El Mundo ${ }^{4}$. Se utiliza un total de 25 datos que se agregan en los seis siguientes grupos: I. Demanda universitaria, II. Personal académico, III. Infraestructura y recursos para la enseñanza, IV. Plan de estudios, V. Resultados del proceso de formación, VI. Información de contexto.

Como se puede apreciar, el emisor en este caso es un agente externo al medio universitario, se trata de un medio de comunicación que genera una información integrada, dirigida a jerarquizar por

4 Disponible en http://aula.elmundo.es/aula/especiales/2005/50carreras/index.htm] 
calidad o excelencia. En dicho esfuerzo no escatima criterios y esa pluralidad le permite mostrar indicadores legitimados por la comunidad académica que, a su vez, contribuye a producir un impacto hacia el interior del propio sistema universitario.

Con lo anterior me permito sugerir que la emisión de rankings desde fuera del sistema de la educación superior en ningún caso constituye un obstáculo para integrarse válidamente como un elemento dinamizador. Es decir, el grado de subjetividad o arbitrariedad que se pueda objetar a los mismos no dice relación, como se dijo, con los agentes que emiten tales evaluaciones, sino con la metodología y variables consideradas.

\section{Universidades, carreras y sedes}

En el caso de Chile una prevención metodológica de la mayor importancia es distinguir entre universidades, carreras y sedes. La proliferación de sedes y carreras que permite la legislación a todas las instituciones representa una amenaza para la validez de la información que se entrega acerca de una universidad. Como se sabe, una vez que las universidades privadas no tradicionales, esto es, las que surgen a partir de 1981, obtienen la certificación de autonomía por parte del Consejo Superior de Educación (CSE), pueden crear todo tipo de carreras e instalar sedes a lo largo del país sin limitación alguna. La Ley Orgánica Constitucional de Enseñanza (LOCE) no otorga facultades de control o supervisión alguna al mencionado Consejo, pues el artículo 57 lo que autoriza es la cancelación de la personalidad jurídica de la institución por causales genéricas, atribución que debería ejercer el Ministerio de Educación. Sin entrar en mayores detalles, cabe decir que, hasta ahora, las instituciones han contado con la más amplia libertad para crear sedes y carreras, que la autoridad administrativa o ha tolerado o se ha inhibido por estimar que carece de las atribuciones para impedir esta proliferación. El mismo fenómeno de expansión descontrolada se ha vivido en universidades públicas, sin que la autoridad administrativa haya ejercido control alguno ni haya aplicado medidas restrictivas o correctoras. 
Lo anterior ha provocado una inquietante heterogeneidad en la calidad ofrecida para una carrera por una misma universidad, pues ello depende del número de sedes en que se imparta. Del mismo modo, una institución puede lograr ciertos niveles de calidad para algunas de sus carreras, pero en otras hallarse todavía en niveles precarios. En consecuencia, considerar a las instituciones como un todo puede resultar muy engañoso para el usuario poco ilustrado.

\section{Conclusiones}

Los rankings constituyen un elemento que forma parte del sistema de educación superior, en cuanto pueden actuar sobre las expectativas de los usuarios y las respuestas de las instituciones, cuya influencia puede llegar a modificar o intervenir en el modelo de universidad vigente en una sociedad dada.

La independencia e imparcialidad de las evaluaciones son condiciones de posibilidad del valioso efecto social que pueden tener sobre el sistema de educación superior, pero éstas se logran no en razón de quien las emite, sino de las metodologías que utilizan.

Al margen de la calidad pública o privada de los agentes emisores, lo determinante es que los rankings se basen en criterios y metodologías reconocidos por las comunidades académicas respectivas.

Las jerarquizaciones de calidad requieren de una pluralidad de criterios y datos cuantificables que deben atender a hechos diferenciados, no implicados entre sí. En la misma medida, los de prestigio o reputación pueden resultar procedimientos más inseguros como descriptores de calidad o excelencia, si no integran una pluralidad de criterios de evaluación.

Los rankings y los procesos de acreditación presentan una relación compleja. Cumplen una función distinta; sin embargo, no pueden conducir a resultados asimétricos, ya que ambos 
procedimientos de evaluación deben producir información validada conforme a criterios reconocidos y aceptados por la comunidad académica. Parece un error eximir a los rankings del rigor y de la diversidad de criterios que supone un juicio sobre calidad de la educación superior. Una tabla de posiciones de prestigio o calidad tiene por objeto actualizar información por periodos más cortos de tiempo que los considerados en un proceso de acreditación, pero no puede apartarse de un núcleo básico de antecedentes sin el cual puede hacer más opaco el sistema de educación superior.

Recibido: 10 de octubre de 2006 Aceptado: 24 de octubre de 2006 